\title{
An Authentication Protocol with Anti-Counterfeit for RFID Communication System
}

\author{
Umesh Kumar Garg \\ M.Tech(CS) \\ Govt. Engg. College \\ Ajmer
}

\author{
Harshad Panwar \\ M.Tech(CS) \\ Govt. Engg. College \\ Ajmer
}

\author{
Neetu Sharma \\ Asst. Professor \\ Govt. Engg. College \\ Ajmer
}

\begin{abstract}
RFID, in today's scenario is playing a vital role in multiple domains, and has become an inevitable tool to manage inventory, animal husbandry, and substituting barcode technology. We see its use at metro stations, so it shows multifaceted use making it an umbrella technology which is cost efficient too. In context to mobile readers, the security of communication channel between reader and backend server is compromised; the same also applies on tag reader communication so prevention of illegal reader and tags to enter the system is at most necessary. Also, counterfeiters have become a global phenomenon cause a threat to security. This paper aims to propose an authentication protocol to check the illegal tag and reader that breaches the system. Hash based function and public key encryption techniques are implemented on authentication protocol.

\section{General Terms}

$$
\mathrm{E}_{\mathrm{A}}(\mathrm{Msg})=>
$$

Security Algorithm.

$$
\mathrm{EP}_{\mathrm{A}}(\mathrm{Msg})=>
$$

\section{Keywords}

Authentication, Anti-counterfeit, public-key encryptipß ${ }_{\mathrm{A}}(\mathrm{Msg})=>$
\end{abstract}

\section{INTRODUCTION}

Radio frequency identification (RFID) is a wireless technology used for identification of living beings and objects[1-3]. RFID may be viewed as a means of labeling objects replacing the traditional technologies AIDC like barcode[5] etc. Inventory management in big firm, cattle tagging in European countries and ticketing system at metro stations are some uses of RFID system. It comprises tag, reader and backend servers.

Since, the communication between tag and reader is always wireless and we also assume that the communication between reader and back-end server (mobile reader) is wireless also. So, this wireless communication which is increasing chances of security breach by an illegal or unauthorized entity.

Although hash based protocols[5] are enable to protect the RFID tag-reader communication and also cryptographic based protocol are very useful to protect the system, But when we talk about the mobile reader then the adversary may be a reader or back-end server and that time these protocols are unable to provide the security between tag and reader. So we propose a new efficient and scalable protocol based on both hashing and public key encryption technique, which assures us to protect the system from counterfeiters

In this proposed protocol, When entities communicate to each other, every time they authenticate to each other and after that they are able to transfer the message. And we use time stamp to protect the system with replay attack and use double public key encryption in which sender encrypt the message with senders private key and again encrypt the message with receiver's public key. So that they assures that the message is send by an authenticate entity. Since, hash based function is one way coding, so it protect the message from adversary and counterfeiters.

\section{PROPOSED PLAN}

We create a new scalable, untraceable and anti-counterfeit authentication protocol rooted on hash function and public key encryption technology to provide the security in RFID tag-reader communication process. The proposed protocol uses following notation:

TSP $\quad \Rightarrow \quad$ Time Stamp

Public key of A is used for

Message (Msg) Encryption,

Private key of A is used for Message Encryption,

Public key of A is used for Message (Msg) Decryption, Private key of A is used for Message (Msg) Decryption,

$\begin{array}{lllll}\Rightarrow & \text { Secret } & \text { key shared by Reader and } \\ \text { Tag } & & & \\ \text { PIN } \Rightarrow & \text { Password shared by Tag and } \\ \text { Backend Server, } & & & \\ \mathrm{ID}_{\mathrm{i}} \Rightarrow & \mathrm{i}^{\text {th }} \text { tag identification, } & \\ \mathrm{h}(\mathrm{Msg}) \Rightarrow & \text { Hash code of Message. }\end{array}$

Our key opinion is to use a secret key with two-way authentication and use public key encryption to prevent from counterfeiters and time stamp is used to prevent from replay attack without implementing time clock in tag $\mathrm{T}$. In this protocol we have taken following assumptions:

Tag T contains ID, KEY, PIN, TSP last $_{\text {. }}$

The reader R contains only KEY shared with tag $\mathrm{T}$.

Backend server contains $\mathrm{ID}_{\mathrm{i}}, \mathrm{h}\left(\mathrm{ID}_{\mathrm{i}}\right)$, EPC, $\mathrm{ID}_{\text {last }}, \mathrm{h}\left(\mathrm{ID}_{\text {last }}\right)$.

\section{Protocol Description}

Step I Firstly, reader R activates and gets the time stamp TSP from its time stamp information then reader calculate the message M1 with hash function and then encrypt this message and TSP with tag's public key and send it to tag T. 
$\mathrm{M} 1=\mathrm{h}(\mathrm{KEY}, \mathrm{TSP})$

$\mathrm{B} 1=\mathrm{E}_{\mathrm{T}}[\mathrm{M} 1, \mathrm{TSP}]$

Send B1 to tag T

Step II After receiving the message B1, tag T decrypts this with its private key and compare time stamp TSP with $\mathrm{TSP}_{\text {last }}$. If TSP is greater, then tag T calculates the message M2 with its KEY and TSP and compares M1 with M2, otherwise discard the message, if they are same, tag authenticates the reader by forwarding self's ID.

$\mathrm{DP}_{\mathrm{T}}[\mathrm{B} 1]=[\mathrm{M} 1, \mathrm{TSP}]$

If, $\mathrm{TSP}>\mathrm{TSP}_{\text {las }}$

Then, $\mathrm{M} 2=\mathrm{h}(\mathrm{KEY}, \mathrm{TSP})$

If $\mathrm{M} 1==\mathrm{M} 2$, then

Send $\underline{B} 2=E_{R}\left[h\left(I D_{i}\right), T S P\right]$ to Reader

Step III After receiving B2, the reader decrypts the message B2 with its private key and compares the TSP with previous TSP (sent by reader in step I). If found equal then reader calculates B3 with two-way encryption with public key of server and private key of reader so that the backend server can check the authentication of the reader.

$\mathrm{DP}_{\mathrm{R}}[\mathrm{B} 2]=\mathrm{h}\left(\mathrm{ID}_{\mathrm{i}}\right)$, TSP

If, $\operatorname{TSP}($ received $)==\operatorname{TSP}($ sent $)$

Then send $\mathrm{B} 3=\mathrm{E}_{\mathrm{S}}\left[\mathrm{EP}_{\mathrm{R}}\left[\mathrm{h}\left(\mathrm{ID}_{\mathrm{i}}\right), \mathrm{TSP}\right]\right]$

Step IV After receiving B3 server decrypts message B3 with its private key and reader's public key. If successful then server find $\mathrm{h}\left(\mathrm{ID}_{\mathrm{i}}\right)$ in its database and extracts PIN to calculate message M3. If ID is not present in its database then it checks $h\left(\mathrm{ID}_{\text {last }}\right)$ and updates database with new value of ID. Now message M3 is encrypted and sent to reader.

$\mathrm{DP}_{\mathrm{s}}\left[\mathrm{D}_{\mathrm{R}}[\mathrm{B} 3]\right]=\mathrm{h}\left(\mathrm{ID}_{\mathrm{i}}\right), \mathrm{TSP}$

Find ID in database and extract PIN.

M3 $=\mathrm{h}(\mathrm{ID}, \mathrm{PIN})$

Send $A 4=\underline{E}_{R}\left[E_{S}[M 3]\right.$ to reader

And update $\mathrm{ID}_{\mathrm{i}+1}=\mathrm{h}\left(\mathrm{ID}_{\mathrm{i}}, \mathrm{PIN}, \mathrm{TSP}\right)$

Step $\mathbf{V}$ After receiving the message A4, reader decrypts it by private key and server's public key so that reader assures that backend server is not malicious followed by encryption of same message with its private key and tag's public key.

$\mathrm{DP}_{\mathrm{R}}\left[\mathrm{D}_{\mathrm{S}}(\mathrm{B} 4)\right]=\mathrm{M} 3$

Send $\mathrm{A} 5=\mathrm{E}_{\mathrm{T}}\left[\mathrm{EP}_{\mathrm{R}}[\mathrm{M} 3, \mathrm{TSP}]\right]$ to tag $\mathrm{T}$

Step VI After receiving the message A5, tag decrypts it by private key and server's public key. Then compare the TSP with previous TSP. if both are equal then tag assures that the message is sent by the authorized reader and then it calculate the M4 by its ID and PIN, and compare this to received M3. If this found same then the tag assures that the server is legal and update its ID value and $\mathrm{TSP}_{\text {last }}$.

$\mathrm{DP}_{\mathrm{T}}\left[\mathrm{D}_{\mathrm{R}}[\mathrm{A} 5]\right]=\mathrm{M} 3, \mathrm{TSP}$

If $\operatorname{TSP}($ Before $)=\mathrm{TSP}($ Latest $)$
Then calculate M4 $=\mathrm{h}(\mathrm{ID}, \mathrm{PIN})$

If $\mathrm{M} 4==\mathrm{M} 3$

Then Authentication Successful

$\mathrm{ID}_{\mathrm{i}+1}=\mathrm{h}\left(\mathrm{ID}_{\mathrm{i}}, \mathrm{PIN}, \mathrm{TSP}\right)$

$\mathrm{TSP}_{\text {last }}=\mathrm{TSP}$

\section{ANALYSIS}

I. De-synchronization:

Proposed plans will remove de-synchronization problem as

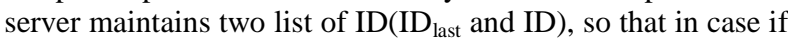
message gets corrupt, we can retrieve it back from ID $_{\text {last }}$.

II. Anti-counterfeit issue:

Since RFID chip is unique which makes it impossible to create a dummy chip with same number. So eavesdropper cannot make copy of same information and thus our purpose is solved.

\section{Forward secrecy:}

Since hashing is one-way encoding scheme so that when we update $\mathrm{ID}_{\mathrm{i}}$ to $\mathrm{ID}_{\mathrm{i}+1}$ there is no way to generate initial information which was hashed i.e. $\mathrm{ID}_{\mathrm{i}+1}$ cannot produce $\mathrm{ID}_{\mathrm{i}}$.

\section{Un-traceability:}

Public key encryption and time stamp at either side make is difficult to trace communication among the entities in the system.

\section{Spoofing:}

The unauthorized copying of RFID tag information that includes its ID and retransmitting it back to reader in lieu to fool it can be checked by public key encoding that is evident in the proposed plan.

\section{Item privacy:}

Private and public key encryption leads to dual encoding and decoding of data making it nearly impossible to disturb the privacy of network.

\section{Replay attack:}

Time stamp plays the role here as every time when information is received by an entity it checks the time stamp to its previous time stamp, so that it can easily trace an attack if a mismatch occurs.

\section{CONCLUSION \& FUTURE WORK}

Earlier work in this field have tried to provide a genuine solution from malicious attack, our proposed work is an effort in the same direction, as here the use of hash function and public-key encryption with time stamping on either side of a two way communication is an effort to remove minimal chances of attack on wireless communication to the fullest.

In context to future work it would be right to emphasize the issue of time complexity, the next step to evaluate time function and minimize it to make system more efficient in real time scenario. 


\section{REFERENCE}

[1] R. Koh, E. W. Schuster, I. Chackrabarti, and A. Bellman, "Securing the Pharmaceutical Supply Chain," White Paper MIT-AUTOID-WH- 021, Auto-Id Center MIT, Cambridge, Ma 02139-4307, USA, 2003, Available at http://www.mitdatacenter.org/MIT-AUTOIDWH021. pdf.

[2] J. Kim, D. Choi, I. Kim, and Kim H., "Product authentication service of consumer's mobile RFID device," IEEE 10th International Symposium on Consumer Electronics, 2006, pp. 1-6.

[3] J. Kim and H. Kim, "A wireless service for product authentication in mobile RFID environment," The 1st International Symposium on Wireless Pervasive Computing, 2006, 5 pages.
[4] Chin-Ling Chen, Yu-Yi Chen, Tzay-Farm Shih, Tsungm-Min Kuo, "An RFID Authentication and Anticounterfeit Transaction Protocol" 2012 International Symposium on Computer, Consumer and Control, pp. 419-422.

[5] Swimpy Pahuja, Sandeep Negi, Ankit Verma, Pulkit Rathi, Nidhi Narang, Rakesh Chawla, "An Authentication Protocol for secure tag-reader communication" 2012 IEEE Students' Conference on Electrical, Electronics and Computer Science.

[6] MEI Shuang, YANG Xiao-long, “An Efficient Authentication Protocol for Low-Cost RFID System in the Prensence of Malicious Readers" $20129^{\text {th }}$ International Conference on Fuzzy System and Knowledge Discovery(FSKD 2012),pp. 2111-2114

[7] Paolo D'Arco and Alfredo De Santis, "On ultralightweight RFID Authentication Protocols", IEEE Transactions on Dependable and Secure Computing, vol. 8, No.4, July/August 2011. 